\title{
Correction to: Viral integration drives multifocal HCC during the occult HBV infection
}

Xiao-Ping Chen ${ }^{1 \dagger}$, Xin Long ${ }^{1+}$, Wen-long Jia ${ }^{2+}$, Han-Jie $\mathrm{Wu}^{3+}$, Jing Zhao ${ }^{4}$, Hui-Fang Liang ${ }^{1}$, Arian Laurence ${ }^{5}$, Jun Zhu', Dong Dong ${ }^{7}$, Yan Chen ${ }^{1}$, Long Lin' ${ }^{1}$, Yu-Dong Xia ${ }^{1}$, Wei-Yang Li ${ }^{1}$, Gui-Bo Li ${ }^{8}$, Zhi-Kun Zhao ${ }^{9}$, Kui Wu', Yong Hou ${ }^{1}$, Jing-Jing Yu ${ }^{1}$, Wei Xiao ${ }^{1}$, Guo-Ping Wang ${ }^{10}$, Peng-Cheng Zhu ${ }^{10}$, Wei Chen ${ }^{1}$, Ming-Zhou Bai ${ }^{1}$, Yi-Xing Jian ${ }^{1}$, Karsten Kristiansen ${ }^{4}$ and Qian Chen ${ }^{11^{*}}$

Correction to: J Exp Clin Cancer Res (2019) 38:261 https://doi.org/10.1186/s13046-019-1273-1

In the original publication of this article [1], Fig. 6 is wrong and the updated figure is shown below.

The authors sincerely apologize for the inconvenience caused to the readers.

\section{Author details}

${ }^{1}$ The Hepatic Surgery Centre at Tongji Hospital, Tongji Medical College, HUST; Hubei Province for the Clinical Medicine Research Center of Hepatic Surgery, Key Laboratory of Organ Transplantation, Ministry of Education and Ministry of Public Health, Wuhan 430030, China. ${ }^{2}$ Department of Computer Science, City University of Hong Kong, Hong Kong, People's Republic of China. ${ }^{3}$ School of Biomedical Engineering, University of Technology Sydney, Sydney, NSW2007, Australia. ${ }^{4}$ Department of Biology, Laboratory of Genomics and Molecular Biomedicine, University of Copenhagen, Universitesparken 13, 2100 Copenhagen, Denmark. ${ }^{5}$ The Newcastle upon Tyne Hospitals NHS Foundation Trust at Freeman Hospital, Newcastle, UK. ${ }^{6}$ Department of Genetics and Genomic Sciences, Icahn Institute of Genomics and Multiscale Biology, Icahn School of Medicine at Mount Sinai, 1425 Madison Avenue, New York, NY, USA. 'Laboratory of Molecular Ecology and Evolution, Institute of Estuarine and Coastal Research, East China Normal University, Shanghai, China. ${ }^{8}$ School of Bioscience and Bioengineering at South China University of Technology, Guangzhou, China. ${ }^{9}$ School of Biological Science and Medical Engineering at Southeast University, Nanjing, China. ${ }^{10}$ The Department of Pathology at Tongji Hospital, Tongji Medical College, HUST, Wuhan 430030, China. ${ }^{11}$ The Division of Gastroenterology, Department of Internal Medicine at Tongji Hospital, Tongji Medical College, Huazhong University of Science and Technology (HUST), Wuhan 430030, China.

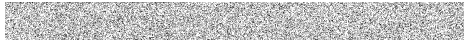

Reference

1. Chen XP, et al. Viral integration drives multifocal HCC during the occult HBV infection. J Exp Clin Cancer Res. 2019;38:261.

The original article can be found online at https://doi.org/10.1186/s13046019-1273-1

*Correspondence: chenqian201579@yahoo.com

${ }^{+}$Xiao-Ping Chen, Xin Long, Wen-long Jia and Han-Jie Wu contributed equally to this work.

${ }^{11}$ The Division of Gastroenterology, Department of Internal Medicine at Tongji Hospital, Tongji Medical College, Huazhong University of Science and Technology (HUST), Wuhan 430030, China

Full list of author information is available at the end of the article

(c) The Author(s). 2019 Open Access This article is distributed under the terms of the Creative Commons Attribution 4.0 International License (http://creativecommons.org/licenses/by/4.0/), which permits unrestricted use, distribution, and reproduction in any medium, provided you give appropriate credit to the original author(s) and the source, provide a link to the Creative Commons license, and indicate if changes were made. The Creative Commons Public Domain Dedication waiver (http://creativecommons.org/publicdomain/zero/1.0/) applies to the data made available in this article, unless otherwise stated. 


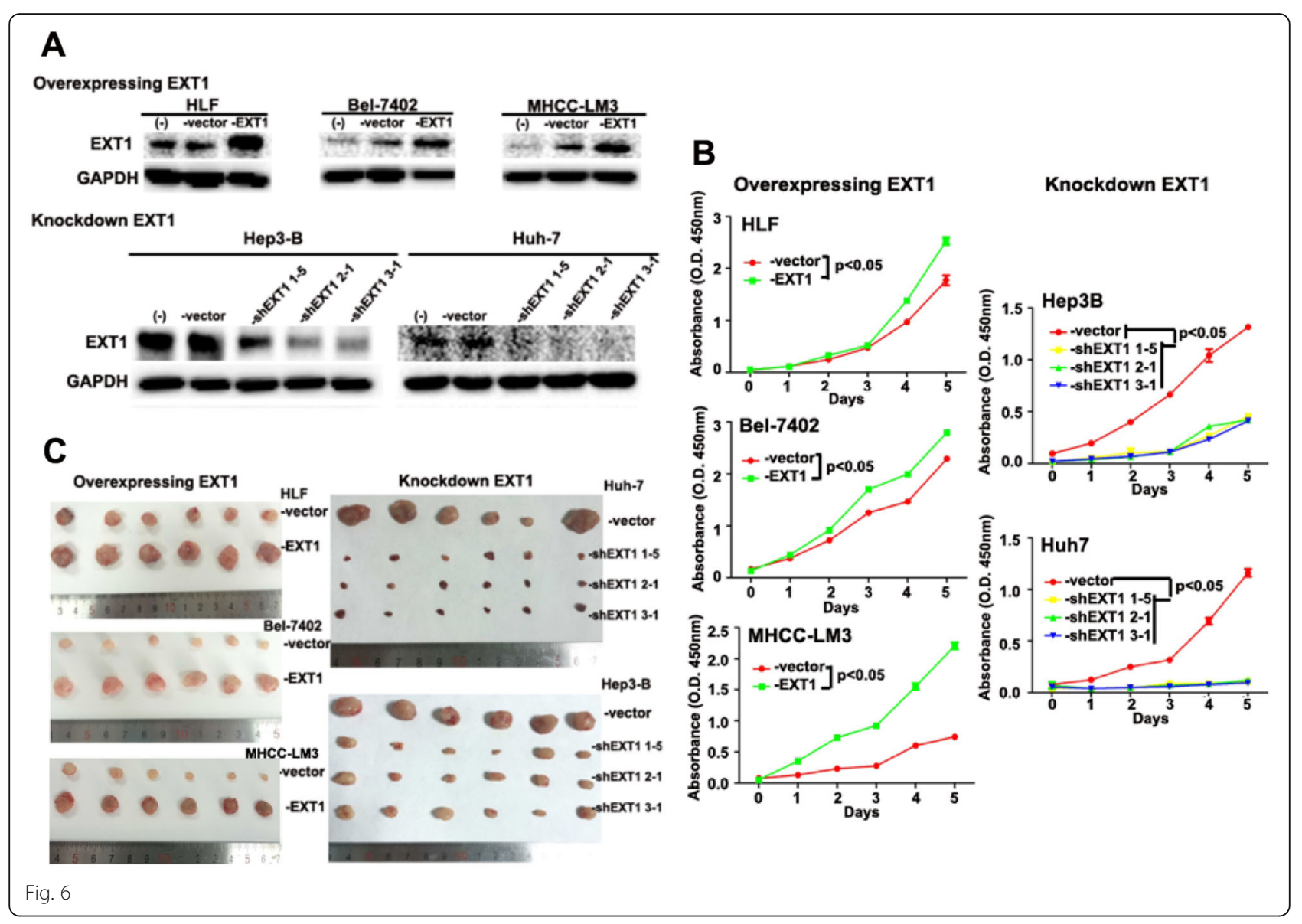

